\title{
Love to Win or Hate to Lose? Asymmetry of Dopamine D2 Receptor Binding Predicts Sensitivity to Reward versus Punishment
}

\author{
Rachel Tomer $^{1 *}$, Heleen A. Slagter ${ }^{1 * *}$, Bradley T. Christian ${ }^{1}$, \\ Andrew S. Fox ${ }^{1}$, Carlye R. King ${ }^{1}$, Dhanabalan Murali ${ }^{1}$, Mark A. Gluck ${ }^{2}$, \\ and Richard J. Davidson ${ }^{1}$
}

\begin{abstract}
Humans show consistent differences in the extent to which their behavior reflects a bias toward appetitive approach-related behavior or avoidance of aversive stimuli [Elliot, A. J. Approach and avoidance motivation. In A. J. Elliot (Ed.), Handbook of approach and avoidance motivation (pp. 3-14). New York: Psychology Press, 2008]. We examined the hypothesis that in healthy participants this motivational bias (assessed by selfreport and by a probabilistic learning task that allows direct comparison of the relative sensitivity to reward and punish-
\end{abstract}

\section{INTRODUCTION}

In everyday life, humans constantly choose actions based on the balance between the desire for pleasure and aversion to punishment. Research has shown that there are consistent differences between individuals in the extent to which their behavior reflects a bias toward appetitive approach-related behavior or avoidance of aversive stimuli. Such individual differences in motivational bias have also been documented within a variety of other species (Jones \& Gosling, 2008), influence a wide range of social and personal behaviors (Elliot, 2008), and extreme imbalance between these two motivational tendencies is thought to underlie some forms of psychopathology (Schutter \& Van Honk, 2005).

One of the major biological theories of individual differences in motivational bias, Gray's Reinforcement Sensitivity Theory (Gray, 1981), describes two systems: the behavioral activation system (BAS), which activates behavior toward incentives, and the behavioral inhibition system (BIS), which guides behavior in response to aver-

\footnotetext{
${ }^{1}$ University of Wisconsin, ${ }^{2}$ Rutgers University-Newark

* Permanent address: Department of Psychology, University of Haifa, Mount Carmel, Haifa 31905, Israel.

** Present address: Department of Psychology and Cognitive Science Center Amsterdam, University of Amsterdam, Weesperplein 4, 1018 XA Amsterdam, the Netherlands.
}

ment) reflects lateralization of dopamine signaling. Using [F-18] fallypride to measure D2/D3 binding, we found that self-reported motivational bias was predicted by the asymmetry of frontal D2 binding. Similarly, striatal and frontal asymmetries in D2 dopamine receptor binding, rather than absolute binding levels, predicted individual differences in learning from reward versus punishment. These results suggest that normal variation in asymmetry of dopamine signaling may, in part, underlie human personality and cognition. sive stimuli. Tonic activation levels of these systems are thought to index stable individual differences in motivational bias, and the relative strength of the BAS and BIS systems has been linked to differences in frontal cortex activation, as reflected in EEG power (Sutton \& Davidson, 1997). Specifically, greater left than right frontal activation has been associated with relatively stronger approach motivation (higher BAS activity), whereas the opposite pattern of frontal activation was related to predominant avoidance motivation (higher BIS activity).

The association between approach/avoidance motivation and asymmetric activation of frontal brain regions has been studied in a large number of EEG and neuroimaging studies. A meta-analysis of 65 PET and fMRI studies (Wager, Phan, Liberzon, \& Taylor, 2003) concluded that approach-related activations show a trend toward leftlateralization in the frontal cortex, whereas withdrawal activations showed bilateral frontal distribution and right lateralization in the BG. A similar finding was reported by a meta-analysis of 106 PET and fMRI studies of human emotion (Murphy, Nimmo-Smith, \& Lawrence, 2003), reporting greater left-sided activity for approach emotions but symmetrical activity associated with negative/withdrawal emotions. A recent review of EEG studies (Harmon-Jones, Gable, \& Peterson, 2010) concluded that much support exists for the association of approach motivational processes with greater left than right frontal activity. These authors also found association of withdrawal motivation 
processes with greater right as compared with left frontal activity. Thus, although much work is still required to clarify to what extent subregions within the frontal cortex show associations with approach/avoidance tendencies and whether asymmetries in brain regions other than the frontal cortex are also related to differential sensitivity to approach versus avoidance, the existing literature provides evidence supporting associations between frontal activation asymmetries and motivational bias. However, little is known about the neurochemistry underlying individual differences in approach and avoidance motivation. This study examined the hypothesis that lateralization of tonic dopamine (DA) activity contributes to individual differences in motivational bias. DA neurons encode motivational value (Bromberg-Martin, Matsumoto, \& Hikosaka, 2010), and DA plays an important role in energizing behavior in preparation for response to appetitive as well as aversive stimuli (Robbins \& Everitt, 2007; Salamone, Correa, Farrar, \& Mingote, 2007). It is therefore noteworthy that the development of frontal cortical asymmetries (that are associated with differential BAS vs. BIS activity) has been attributed to input from asymmetric subcortical neurochemical systems, including dopaminergic systems (Trevarthen, 1996). Asymmetric tonic DA activity may thus contribute to the asymmetric level of tonic prefrontal activation and thereby modulate motivational bias. Consistent with this idea, in animals, individual differences in dopaminergic asymmetry predict a wide range of behaviors related to approach and avoidance motivation, including emotional reactivity (Thiel \& Schwartling, 2001) and drug sensitivity (Carlson \& Stevens, 2006). Asymmetries within the DA system have also been reported in the healthy human brain (Vernaleken et al., 2007; Van Dyck et al., 2002; Laakso et al., 2000; Hietala et al., 1999; Larisch et al., 1998). Yet, the relevance of asymmetric dopamine activity to differences in such fundamental behaviors in humans has not been studied to date. This knowledge may provide important clues for understanding how variability in dopamine asymmetry impacts behavior in healthy individuals as well as in pathological states.

On the basis of the above summarized literature and previous studies of patients with Parkinson's disease (Tomer \& Aharon-Peretz, 2004) as well as healthy individuals (Tomer, Goldstein, Wang, Wong, \& Volkow, 2008; Sutton \& Davidson, 1997), we hypothesized that the direction and magnitude of asymmetries in dopaminergic function in striatal and frontal brain regions in healthy participants would predict individual differences in motivational bias toward positive outcomes or away from negative outcomes. This bias should be revealed not only by self-report (the BIS/BAS measure employed in previous studies) but also in the performance of tasks that assess differences in the sensitivity to reward and punishment. To test this hypothesis, we measured baseline dopamine D2/D3 receptor binding with PET and the high-affinity radioligand [F-18]fallypride. The basal striatal dopamine concentration of 5-10 nM is sufficient to tonically stimulate D2 receptors in the high-affinity state (Schultz, 2007). We therefore reasoned that D2/D3 receptor binding at rest (while participants were not presented with any stimulation and not performing any task), possibly reflecting altered DA levels from chronic adaptation, can serve as an index for this enabling modulatory role of DA on postsynaptic neurons in the frontal cortex. Good reproducibility of D2 receptor binding in healthy volunteers (using [F-18]fallypride) over a period of 4-6 weeks was reported in all brain regions (Mukherjee et al., 2002), and therefore, asymmetries in baseline D2 receptor binding may serve as a trait index of individual differences in dopamine asymmetry. Individual differences in self-reported motivational preferences were evaluated using the BIS/BAS questionnaire (Carver \& White, 1994). To assess differential sensitivity to appetitive versus aversive stimuli, participants performed a probabilistic learning task that allows direct comparison of the relative sensitivity to reward and punishment (Bódi et al., 2009).

\section{METHODS \\ Participants}

Fourteen undergraduate students (nine women, age range $=19-29$ years, average age $=20.2 \pm 2.9$ years, all right-handed) participated in this study. Only healthy participants without history of developmental disorders, head trauma, psychiatric or neurological disease, and current or previous drug use were included. All had normal or corrected-to-normal visual acuity. Participants were compensated for participation. The study was approved by the institute's ethics committee, and all participants gave written informed consent.

\section{Experimental Design and Procedure}

Data were collected in three sessions, separated by $1-$ 4 weeks: Behavioral data were collected in the first session in which participants completed the BIS/BAS questionnaire (Carver \& White, 1994) and performed a reward versus punishment learning task (Bódi et al., 2009). PET imaging to measure D2/D3 receptor availability was conducted in the second session, and MRI scanning to acquire anatomical brain images was conducted in the third session.

\section{BIS/BAS Questionnaire (Carver \& White, 1994)}

This self-report measure was designed to assess individual differences in general strength of the BAS and the BIS. In general, the BAS scale assesses the tendency to experience strong positive affect or behavioral approach when specific goal-oriented situations are encountered. The BIS scale assesses the tendency to experience strong negative affect or behavioral inhibition when perceived threats are encountered. These measures were shown to have high internal consistency and good test-retest 
reliability over an 8-week period. The questionnaire consists of 20 items ( 7 items constitute the BIS subscale, and 13 items constitute the BAS scale).

\section{The Reward versus Punishment Learning Task (Bódi et al., 2009)}

In this computer-based probabilistic classification task, participants view one of four images and are asked to guess whether it belongs to category A or category B. On reward-learning trials (with stimuli S1 [80\% category A] or S2 [80\% category B]), correct answers are rewarded with positive feedback and gain of 25 points; incorrect answers receive no feedback. On punishment-learning trials (with stimuli S3 [80\% category A] or S4 [80\% category B]), incorrect answers are punished with negative feedback and loss of 25 points; correct answers receive no feedback. The task consists of four blocks of 40 trials each. Within a block, trial order is randomized. Trials are separated by a 2 -sec interval, during which time the screen is blank. Within each block, each stimulus appears 10 times, 8 times with the more common outcome (e.g., category "A" for S1 and S3 and "B" for S2 and S4) and 2 times with the less common outcome. On each trial, the computer records whether the participant made the optimal response (i.e., category A for S1 and S3 and category B for S2 and S4) regardless of actual outcome. This task has been shown to be sensitive to dopamine functioning in patients with Parkinson disease (Bódi et al., 2009).

\section{Imaging Data Acquisition and Analysis}

\section{MRI Acquisition}

Anatomical brain images were acquired on a 3-T GE Signa scanner, which is equipped with high-speed gradients and a whole-head transmit-receive quadrature birdcage headcoil (GE Medical Systems, Milwaukee, WI). Anatomical scans consisted of a high-resolution 3-D T1-weighted inversion recovery fast gradient-echo image (inversion time $=$ $600 \mathrm{msec}, 256 \times 256$ in-plane resolution, field of view $=$ $240 \mathrm{~mm}, 124 \times 1.1 \mathrm{~mm}$ axial slices) and a T2-weighted fast spin echo image $(256 \times 256$ in-plane resolution, field of view $=240 \mathrm{~mm}, 81 \times 2 \mathrm{~mm}$ sagittal slices).

\section{Radiochemical Synthesis}

The synthesis of [F-18]fallypride was carried out using previously reported methods (Mukherjee, Yang, Das, \& Brown, 1995). The final sterile $0.9 \%$ saline solution of [F-18] fallypride was produced with radiochemical purity greater than 95\% and specific activity of $227 \pm 140 \mathrm{GBq} / \mathrm{umol}$.

\section{PET Acquisition}

The PET data were acquired using a Siemens HR+ PET scanner in 3-D mode (septa retracted). Participants were asked to abstain from smoking, eating, or drinking coffee for at least $4 \mathrm{hr}$ before scanning. They were positioned head first, supine with the cantho-meatal line parallel to the in-plane field of view. The head rests in the scanner head holder extending from the patient bed and held in place by surgical tape placed firmly across the participant's forehead. A 5-min transmission scan was then acquired to correct for the attenuation of the gamma rays within the tissue. The acquisition of the dynamic [F-18] fallypride PET scan was initiated with the injection of radioligand (237 $\pm 43 \mathrm{MBq})$. A 150-min dynamic acquisition was acquired, initiating with the 30 -sec bolus infusion of radiotracer. The time series were binned into six 1-min frames and forty-eight 3-min frames. Following the acquisition of the PET data, the participant was removed from the PET scanner.

\section{Data Processing}

The PET data were reconstructed using a filtered backprojection algorithm with sinogram trimming, axial, and in-plane smoothing ( $4 \mathrm{~mm}$ Gaussian filter) to a voxel size of $1.84 \mathrm{~mm} \times 1.84 \mathrm{~mm} \times 2.43 \mathrm{~mm}$ and corrected for random events, attenuation of annihilation radiation, deadtime, scanner normalization, and scatter radiation. The reconstructed PET time series was then inspected and corrected for head motion during the acquisition of the scan using the SPM2 coregistration (Wellcome Trust Centre for Neuroimaging, www.fil.ion.ucl.ac.uk/ spm) algorithm based on frame to frame coregistration to an early integrated reference image (22-43 $\mathrm{min}$ ), which has been shown to provide adequate performance for D2/D3 dynamic PET studies (Montgomery et al., 2006). The cerebellar time-activity curve was extracted from the PET data based on an ROI drawn on the cerebellar lobes of the early PET data (summation of first $6 \mathrm{~min}$, Frames 1-6). Parametric images of distribution volume ratios (DVRs) were generated using the cerebellar time course to represent the behavior of the radiotracer in brain regions with negligible binding (Mukherjee et al., 2002). The DVR parameter represents an index that is proportional to the concentration of D2/D3 binding sites $\left(B_{\max }\right)$, given by the relationship:

$$
D V R=\left(B_{\max } / K_{\mathrm{D}}\right) f_{\mathrm{ND}}+1
$$

where $K_{\mathrm{D}}$ is the apparent (in vivo) equilibrium dissociation constant and $f_{\mathrm{ND}}$ is the free fraction of radiotracer in the brain tissue (Innis et al., 2007). A multilinear approach was used to generate the DVR estimates using the data starting at $39 \mathrm{~min}\left(t^{*}\right)$ until the end of the acquisition (Ichise, Toyama, Innis, \& Carson, 2002; Logan et al., 1996). The DVR parametric images were spatially coregistered to the same participant's T1-weighted MRI images using the FSL linear registration tool (FLIRT; Jenkinson \& Smith, 2001).

To compare both DA binding and the relative asymmetry of DA binding across participants, we used a novel 
strategy for spatial normalization. T1-weighted MRI images were manually masked to exclude nonbrain tissues. These "skull-stripped" T1-weighteted MRI images for each participant were coregistered to the MNI-152 template packaged with FSL twice, once using the default options and once enforcing a left/right flip during the transformation. The resulting standard space skull-stripped T1weigted MRI images were averaged to create single-subject templates in standard space. Small differences in brain asymmetry were accounted for by performing a nonlinear warp using FSL's nonlinear registration tool (FNIRT; www. fmrib.ox.ac.uk/analysis/techrep/tr07ja2/tr07ja2.pdf) to align each participant's flipped and nonflipped standard space skull-stripped T1-weighted MRI images to their singlesubject template. These transformations were then combined with the DVR to T1-weighted MRI transforms and applied to the DVR images to create both absolute amount and relative asymmetry DA images. To compute a measure of relative asymmetry in binding, DA asymmetry images were created by subtracting the $x$-flipped template-space images from the nonflipped template-space images. Because differences observed in across-subject analyses might result from individual differences in brain anatomy, rather than true differences in DA binding, we assessed the probability of gray matter at each voxel in each participant's brain. Estimates of gray matter probability (GMP) were assessed based on the skull-stripped standard space T1weighted MRIs using FSL's automated segmentation tool (Zhang, Brady, \& Smith, 2001). Similar to the DA asymmetry images, GMP asymmetry images were computed by subtracting the $x$-flipped GMP images from the nonflipped GMP images. This resulted in an estimate of gray matter asymmetry for each voxel and individual separately. Before statistical analyses, all images were blurred using an 8-mm FWHM Gaussian filter to account for potential across-subject differences in anatomy.

\section{Relationship between DA Binding and Motivational Bias}

Our main prediction was that asymmetries in DA binding in the striatum and frontal brain regions would be predictive of individual differences in motivational bias. Therefore, we limited our analyses to voxels within frontal brain regions and the BG. To this end, we first created ROIs based on the Harvard-Oxford structural atlas. These ROIs included the left and right putamen, caudate, pallidus and nucleus accumbens, and all frontal regions (i.e., frontal pole, superior frontal gyrus, middle frontal gyrus, inferior frontal gyrus [pars triangularis and pars opercularis], precentral gyrus, frontal medial cortex, juxtapositional lobule cortex, subcallosal cortex, paracingulate gyrus, cingulate gyrus, OFC, and frontal operculum cortex). Within these ROIs, we examined, for each voxel separately, the association between DA binding asymmetry and motivational bias. We took a voxel-wise approach, rather than an ROI approach, as we did not expect an entire region to show an association, in particular given that our measure is an asymmetry score. To derive a measure for behavioral motivational bias, we calculated a Reward-Punishment score for the relative sensitivity to feedback valence for each participant by subtracting the percent of optimal responses in the negative feedback condition from the percent of optimal responses in the positive feedback condition. A positive score indicates more optimal responses to positive feedback and thus higher sensitivity to reward, whereas a negative score reflects greater sensitivity to punishment. An index of the relative dominance of the self-reported approach versus avoidance tendencies of each participant was calculated by subtracting the $z$-transformed BIS score from the $z$-transformed BAS score. Thus, a positive value indicates a relatively higher BAS score and a preference for goal-approaching behavior relative to the tendency to avoid aversive stimuli, whereas a negative score denotes the opposite preference. This difference score showed a high (.81) test-retest stability over a 5 -month period in a sample of young healthy individuals (Sutton \& Davidson, 1997).

To examine the relationship between these measures of motivational bias and asymmetry in D2-like receptor binding, next, a cross-subject Spearman's correlation was run between Reward-Punishment score or BAS-BIS score and asymmetry in D2-like receptor binding (while controlling for gray matter asymmetry). Only voxels within our frontal and BG ROIs were included in the correlation analysis. Using this threshold, a 10\% difference in receptor-specific D2/D3 binding between hemispheres would correspond to roughly a $2 \%$ difference in total PET signal, which is consistent with the accuracy of the quarterly scanner calibration of our PET scanner (1.8\%) using a radioactive source with similar concentrations to those observed in the extrastriatal regions. For statistical thresholding, a two-step nonparametric permutation approach was used (Nichols \& Holmes, 2002). At the first stage (voxel level), as a first step, gray matter asymmetry was regressed out of D2-like receptor binding asymmetry. Then, participant identity was randomly shuffled, and Spearman's correlation between binding asymmetry and Reward-Punishment score or BAS-BIS score was computed again at each voxel within our ROIs. This was repeated 1000 times, generating a distribution of correlation coefficients at each voxel under the null hypothesis of no relationship between the given score and asymmetry in D2-like receptor binding (while controlling for gray matter asymmetry). Statistical $Z$ values were taken as the normalized distance of the real correlation coefficient compared with the null distribution. Voxels with a $Z$ value greater than $2.6(p<.005)$ were retained as being significant at the voxel level (cf. Tomer et al., 2013; Slagter et al., 2012). In the second stage (cluster level), $Z$ values were computed based on 1 of the 1000 random permutation iterations, and the statistical map was thresholded again. This time, the number of voxels in the largest suprathreshold cluster was stored. This was repeated 500 times, 
Figure 1. Association between asymmetric D2 receptor binding and differential learning from reward versus punishment feedback in a probabilistic classification task. Positive learning score denotes better learning from reward feedback (gain), whereas negative score reflects better learning from punishment feedback (loss). Top: Clusters [MNI coordinates of peak voxel] in the left hemisphere, where higher D2 binding was associated with better reward than punishment learning. Bottom: Scatter plots showing the cross-subject correlation between D2 binding asymmetry and RewardPunishment learning score, separately for the striatal and frontal clusters. Binding asymmetry values are based on the peak voxel within each cluster. For illustrative purposes, the correlation is shown using the nonranked data. Note that we only examined the relationship between binding asymmetry and Reward-Punishment learning for voxels within striatal and frontal ROIs.

generating a distribution of maximum cluster sizes under the null hypothesis. The cluster threshold was defined as the standardized distance from the mean of the maximum cluster distribution corresponding to $p<.01$. To examine whether absolute D2-like receptor binding may also be related to one's motivational bias (measured by the Reward-Punishment score or Zbas-zBIS score), the above procedure was repeated, but with absolute binding (rather than binding asymmetry) as the dependent measure and while controlling for absolute GMP.

\section{RESULTS}

All participants were successful in learning to classify the stimuli based on the feedback they received. However, as expected, participants differed considerably in the degree of relative sensitivity to positive versus negative feedback. This differential sensitivity was revealed very early on, as all participants have established a clear bias toward either positive or negative feedback by the end of the first block, and for 13 of the 14 participants in this study, the bias score remained the same throughout the subsequent blocks. This was reflected in the results of a repeatedmeasures ANOVA (Feedback Valence $\times$ Trial Block), which revealed no main effect of Block $[F(3,39)=1.169, n s]$ and no interaction between Feedback Valence and Block $[F(3$,
Table 1. MNI Coordinates for Clusters Showing Significant Correlations between DA D2-like Receptor Binding Asymmetry and Motivational Bias Scores

\begin{tabular}{llllll}
\multicolumn{2}{l}{ MNI Coordinates } & & & No. of \\
\cline { 1 - 3 }$x$ & $y$ & $z$ & & $t$ & Voxels
\end{tabular}

Reward-Punishment Score

Medial frontal cortex

$\begin{array}{lrrrrr}\text { Precentral gyrus } & -6 & -22 & 50 & 3.6 & \\ \text { Cingulate gyrus } & -2 & -4 & 46 & 3.1 & \\ \text { Cingulate gyrus } & -10 & 20 & 40 & 3.1 & \\ \text { Supplementary } & -10 & 8 & 52 & 3.2 & \\ \quad \text { motor cortex } & & & & & \\ \text { Frontal pole } & -8 & 58 & 32 & 2.8 & 17 \\ \text { Orbitofrontal cortex } & -26 & 38 & -14 & 2.7 & 4 \\ \text { Putamen } & -20 & 12 & -8 & 2.7 & 6\end{array}$

\section{ZBAS-zBIS Score}

Middle frontal gyrus

$\begin{array}{rrrr}-32 & 0 & 50 & 3.1 \\ -14 & -8 & 54 & 2.9\end{array}$

Supplementary

$-14$

$-8$

2.9 
Figure 2. Correlations between $\mathrm{D} 2$ binding asymmetry values in the striatal and frontal clusters shown in Figure 1 .
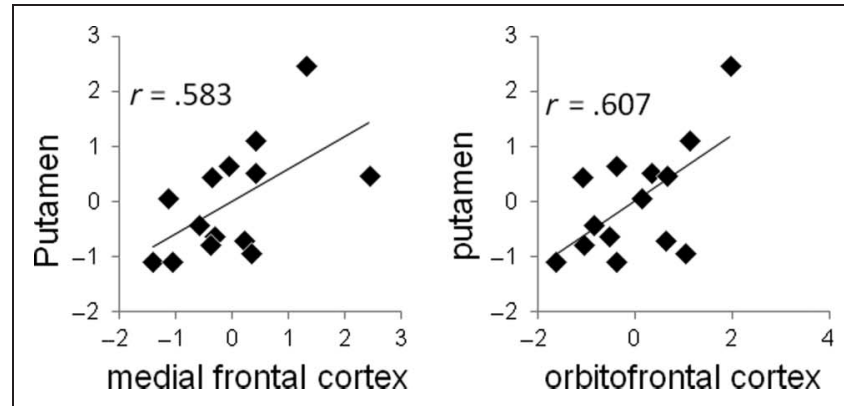

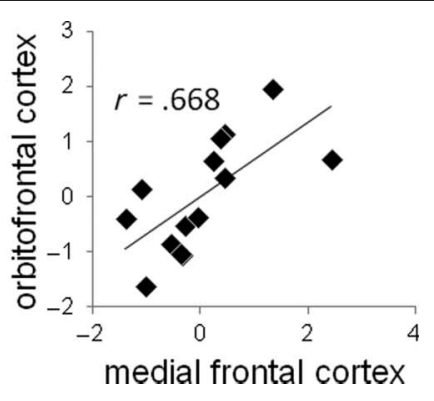

$39)=0.391, n s]$. More importantly and as predicted, the individual bias toward reward or punishment was related to the direction and degree of asymmetry in D2 binding in several striatal and frontal brain regions, such that relatively greater sensitivity to reward was associated with relatively higher $\mathrm{D} 2$ binding in the left hemisphere, whereas increased sensitivity to punishment was associated with the opposite D2 binding asymmetry (Figure 1, Table 1). Specifically, voxel-wise correlation analyses between D2 binding asymmetry and the Reward-Punishment score revealed clusters in the putamen $(x, y, z$ peak coordinates: $-20,12,-8 ; z-\max [12]=2.7, p<.005)$, medial frontal cortex/cingulate gyrus $(x, y, z$ peak coordinates: $-10,20$, $40 ; z$-max $[12]=3.1, p<.005)$, and OFC $(x, y, z$ peak coordinates: $-26,38,-14 ; z-\max [12]=2.7, p<.005)$. In all clusters, higher binding in the left relative to the right hemisphere predicted more optimal responses in the reward feedback condition than punishment feedback condition, whereas the opposite binding asymmetry predicted greater sensitivity to negative feedback. As reported in the Methods section, gray matter asymmetry was regressed out of D2-like receptor binding asymmetry to rule out the effect of anatomical asymmetries. Examination of the correlations between D2 binding asymmetry and anatomical asymmetry for all regions demonstrating a significant relationship between D2 binding asymmetry and our measures of motivational bias (listed in Table 1) revealed that these correlations were not significant (varying between $r=.02$ to $r=.37$, all $p s>.24$ ).

Notably, the asymmetry scores for the peak voxels in the putamen and frontal clusters were positively correlated across individuals (Figure 2), suggesting that baseline asymmetry in both subcortical and frontal regions may provide a similar trait index of individual differences in motivational bias. Importantly, the relative sensitivity to reward versus punishment was not related to absolute values of $\mathrm{D} 2$ receptor binding in individual hemispheres, suggesting that despite differences in the absolute levels of $\mathrm{D} 2$ bindings and lack of correlation between these absolute levels in the striatum and frontal regions (as reported by Cervenka, Varrone, Fransen, Halldin, \& Farde, 2010), the asymmetry between the left and right hemispheres is similar in these different regions, thus underscoring the importance of asymmetric D2 binding as the critical predictor of this motivational bias.

Similarly to the behavioral index of motivational bias, zBAS-zBIS scores also revealed individual differences in the relative strength of the tendency toward appetitive, positive stimuli or away from aversive situations. As predicted on the basis of previous studies reporting association between zBAS-zBIS score and frontal EEG asymmetry (Sutton \& Davidson, 1997), we found a significant correlation between D2 receptor binding asymmetry in the middle frontal gyrus $(x, y, z$ peak
Figure 3. Association between asymmetric D2 receptor binding and self-reported motivational bias. A positive score indicates a relatively higher BAS score and a preference for goal-approaching behavior relative to the tendency to avoid aversive stimuli, whereas a negative score denotes the opposite preference. Left: Cluster [MNI coordinates of peak voxel] in the left hemisphere, where higher D2 binding was associated with BAS > BIS score. Right: Scatter plot

showing the cross-subject correlation between D2 binding asymmetry and zBAS-zBIS score. Binding asymmetry values are based on the peak voxel within this cluster. For illustrative purposes the correlation is shown using the nonranked data. Note that we only examined the relationship between binding asymmetry and motivational bias for voxels within striatal and frontal ROIs. 
Table 2. MNI Coordinates for Clusters outside the Basal Ganglia and Frontal Cortex Showing Significant Correlations between DA D2-like Receptor Binding Asymmetry and Motivational Bias Scores

\begin{tabular}{lccrr}
\hline & \multicolumn{3}{c}{ MNI Coordinates } & \\
\cline { 2 - 4 } & $x$ & $y$ & $z$ & $t$ \\
\hline Reward-Punishment Score & & & & \\
Superior parietal lobule & -16 & -54 & 52 & 2.9 \\
Lingual gyrus & -8 & -62 & 8 & 2.9 \\
& & & & \\
ZBAS-zBIS Score & & & & \\
Inferior temporal gyrus & -58 & -48 & -12 & 3.1 \\
Angular gyrus & 56 & -48 & 36 & 3.0 \\
\hline
\end{tabular}

coordinates: $-32,0,50$; z-max $[12]=3.1, p<.005$; Figure 3) and self-reported motivational bias, such that relatively higher binding in the left hemisphere was associated with relatively higher self-reported BAS score, whereas the opposite binding asymmetry was related to relatively higher BIS scores. Similarly to the behavioral index of motivational bias, self-report bias was not associated with the absolute values of D2 binding in either hemisphere. There was a positive, albeit nonsignificant, correlation between the self-reported bias (zBAS-BIS score) and the behavioral measure of percent optimal responses to Reward-Punishment $(r=.448)$. There were no significant association between D2 receptor binding asymmetry and BIS-BAS bias in any of the clusters that show a relationship between D2 receptor binding asymmetry and reward-punishment feedback bias. Similarly, there was no association between D2 receptor binding asymmetry and reward-punishment feedback bias in any of the clusters with significant association between D2 receptor binding asymmetry and BIS-BAS bias. Thus, in our sample, the task performance and the self-report measure reflect different aspects of motivational bias, but importantly, both aspects are associated with asymmetric availability of D2 receptors.

Whole-brain exploratory analyses, conducted post hoc to determine whether brain regions outside the BG and frontal cortex also showed a relationship between D2-like receptor binding and our measures of motivational bias (following the same statistical approach as for the ROIbased analyses), revealed several additional significant clusters in parietal and temporal cortex (Table 2).

\section{DISCUSSION}

As predicted, we found that individual differences in baseline asymmetric binding to D2-like receptors in the putamen and frontal cortex of healthy participants predict differences in motivational bias, indexed by relatively greater sensitivity to positive, approach-related versus aversive experiences. Specifically, and as expected, our results show that relatively higher D2 receptor binding in the left hemisphere is associated with preference for rewarding events, whereas stronger tendency to avoid aversive outcomes is predicted by relatively higher binding in the right hemisphere. A previous study (Tomer et al., 2008) reported similar associations between D2 binding asymmetry in the putamen and self-reported incentive motivation. However, that study did not include measures of avoidance motivation and was limited to selfreport rather than looking at actual behavioral differences. The current results extend these earlier findings by looking at the motivational bias between approach and avoidance tendencies, evaluating not only self-reported preferences but also relative sensitivity to reward versus punishment in a behavioral task. Importantly, absolute binding values in each hemisphere were not associated with either measure of motivational bias, and asymmetry in D2 receptor binding was not related to absolute levels of approach or avoidance tendencies but predicted the direction and relative strength of the motivational bias. We also regressed out variations in gray matter density so that whatever anatomical asymmetries may have been present did not contribute to our observed findings on D2 binding asymmetries. The current findings thus highlight the utility of the formal assessment of asymmetric signals in human imaging data, because it was only when such asymmetric variation was computed that individual differences in sensitivity to reward vs. punishment were predicted.

The contribution of DA signaling to motivation-related processes is well established (Bromberg-Martin et al., 2010; Berridge, Robinson, \& Aldridge, 2009; Robbins \& Everitt, 2007; Salamone et al., 2007). Although the greatest emphasis has been on phasic responses of DA neurons to reward-related events, a large variety of behavioral functions of postsynaptic striatal and prefrontal neurons depend on tonic stimulation of DA receptors (Schultz, 2007). The current data extend these findings by showing that asymmetries in baseline levels of D2 receptor binding (presumably reflecting tonic DA activity) may contribute to individual differences in motivated behaviors.

As described in the Introduction, differences in motivational bias have been suggested to reflect consistent asymmetrical activation in anterior brain regions. An intriguing possibility is thus that the asymmetry in D2 availability observed in this study contributes to the asymmetric pattern of activation in the frontal lobes and thus to the corresponding individual differences in temperament and behavior, reported by previous studies. In line with this possibility, significant correlations between absolute striatal D2 receptor binding and activity in the OFC and the anterior cingulate gyrus have been observed (Volkow et al., 2001). Remarkably, we found that motivational bias was associated with D2 binding asymmetries in the identical regions, and there were significant positive correlations between the measures of binding asymmetries in 
these regions (Figure 2). Future studies using pharmacological manipulations are necessary to directly relate asymmetries in DA activity to asymmetries in frontal activation and related asymmetries in motivated behaviors in humans.

We found that asymmetric DA availability predicts the degree of relative sensitivity to rewarding versus aversive events as revealed by the number of optimal responses to positive versus negative feedback. Differential effect of D2 receptor stimulation has been shown to affect rewardbased and punishment-based reversal learning differentially, depending on baseline levels of DA synthesis in the dorsal striatum (Cools et al., 2009). This finding, relating individual differences in DA in the dorsal striatum to differential response to reward versus punishment feedback, is consistent with the current results, although in the findings of Cools et al.'s study asymmetric effects may have been masked by the averaged data across the left and right caudate nucleus and putamen. Although performance in similar paradigms have been interpreted as reflecting specific reinforcement learning bias (Bódi et al., 2009; Frank, Seeberger, \& O'Reilly, 2004), we believe that the current association between task performance and DA asymmetry is related to a motivational bias rather than learning, because of the absence of a differential learning rate from the first to the last block, and the finding that practically all participants showed a clear and consistent bias throughout the task. Interestingly, two recent studies (Shiner et al., 2012; Smittenaar et al., 2012) have suggested that DA modulation of performance in a similar task reflects the effect of DA on motivation and cannot be attributed only to reinforcement learning.

We found association between behavioral indices of sensitivity to reward-punishment feedback and asymmetric D2 availability in the putamen. Previous studies have similarly reported an association between D2 receptor availability in the putamen and sensitivity to valenced feedback (Groman et al., 2011; Haber \& Knutson, 2009; Hakyemez, Dagher, Smith, \& Zald, 2008; Zald et al., 2004). A significant correlation between $\mathrm{D} 2$ receptor availability in the putamen and FDG metabolism in the OFC and in the anterior cingulate gyrus was reported by Volkow et al. (2001). This association could reflect dopamine-mediated striatal regulation of orbitofrontal activity by means of striato-thalamo-cortical pathways (Haber, Kim, Mailly, \& Calzavara, 2006). Thus, the asymmetry in D2 receptor binding in the putamen may contribute to the asymmetric pattern of activation in the frontal lobes, resulting in differential sensitivity to reward versus punishment and motivational bias toward approach/avoidance.

Structural and functional asymmetries are evolutionarily ancient traits in vertebrates (Bisazza, Rogers, \& Vallortigara, 1998) that are established during ontogeny and are controlled by genetic as well as environmental factors (Concha, Biaanco, \& Wilson, 2012). Such asymmetries may confer various advantages. Specifically and related to the current study, it was suggested that the existence of paired neural circuits which act differentially to modulate a specific behavioral function may be an evolutionary successful strategy in animal evolution (Alvarez \& Banzan, 2011) and individual differences in the pattern of lateralization may serve the purpose of generating adaptive variation in personality factors (Andrew, 2009). Thus, the current findings suggest that individual differences in DA asymmetry are the underpinnings of individual differences in motivational bias. The molecular mechanisms that are involved in the formation of brain asymmetries are not yet well understood and may include specific genetic polymorphisms (Toga \& Thompson, 2003). Individual differences in various aspects of motivation have been shown to be influenced by genetic factors, and genetic variations in genes controlling dopaminergic function in frontostriatal circuits have been related to approach and avoidance behaviors (for recent reviews, see Frank \& Fossella, 2011; Yacubian $\&$ Buchel, 2009). However, whether these genetic variations also contribute to asymmetric manifestations within the dopaminergic system is currently unknown.

We measured D2/D3 receptor binding, which is an index of receptor availability and fallypride receptor affinity. As such, regional differences in D2/D3 binding could represent differences in D2 receptor density, the equilibrium dissociation constant (Kd), or competing endogenous dopamine. Therefore, it is difficult to determine whether the asymmetry of D2 receptor binding reported here represents asymmetry of receptor density or asymmetry of levels of endogenous dopamine, or both. Further studies are needed to clarify this point. However, the present findings are in line with findings from studies that examined Parkinson's disease patients with asymmetric DA loss. In an earlier study (Tomer \& Aharon-Peretz, 2004), only patients with greater DA loss in the left hemisphere reported reduced novelty seeking (an approach-related behavior), whereas only patients with greater DA deficit in the right hemisphere differed from matched healthy individuals in self-reported harm avoidance (an avoidancerelated behavior). More recently, Porat, Hassin-Baer, Cohen, Markus, and Tomer (2013) reported that patients with relatively greater DA deficit in the left hemisphere exerted less effort to increase gain than to avoid loss, whereas the opposite pattern of effort expenditure was observed by patients with more severe DA deficit in the right hemisphere. Together with the current results, these findings suggest that asymmetric modulation of frontal activity by subcortical DA contributes to differential sensitivity to reward versus punishment and motivational bias toward approach/avoidance.

Our results show that individual differences in striatal and cortical dopamine asymmetries contribute significantly to variability across individuals in important dimensions of personality-approach and avoidance motivation-that influence behavior in a wide range of contexts (Elliot, 2008). Extreme imbalance between these two motivational tendencies has been proposed to underlie some forms of psychopathology. Indeed, enhanced sensitivity to reward 
and reduced sensitivity to punishment was observed in pathological gamblers (van Holst, van den Brink, Veltman, \& Goudiaan, 2010). Aberrations in the response to negative versus positive feedback have also been suggested as characteristic of the impulsive-compulsive disorder that may develop in patients with Parkinson's disease (Ray \& Strafella, 2010). Our findings suggest that aberrant patterns of asymmetry in DA signaling may be involved in the pathophysiology of these states as well as other pathological manifestations of motivated behavior.

\section{Acknowledgments}

This research was supported by grants from the National Institute of Mental Health (MH43454, P50-MH084051) and the Fetzer Institute to R. J. D. and core support from P30-HD003352 (M. Seltzer, PI). M. A. G. was supported by grants from the NIH (NIH/NINDS P50 NS071675-03) and from the Bachmann-Strauss and Dekker Foundations. H. A. S. was supported by a VIDI grant by the Dutch grant agency NWO.

Reprint requests should be sent to Rachel Tomer, Department of Psychology, University of Haifa, Mount Carmel, Haifa 31905 , Israel, or via e-mail: rtomer@psy.haifa.ac.il.

\section{REFERENCES}

Alvarez, E. O., \& Banzan, A. M. (2011). Functional lateralization of the baso-lateral amygdala neural circuits modulating the motivated exploratory behaviour in rats: Role of histamine. Behavioural Brain Research, 218, 158-164.

Andrew, R. J. (2009). Origins of asymmetry in the CNS. Seminars in Cell and Developmental Biology, 20, 485-490.

Berridge, K. C., Robinson, T. E., \& Aldridge, J. W. (2009). Dissecting components of reward: "Liking," "wanting" and learning. Current Opinion in Pharmacology, 9, 65-73.

Bisazza, A., Rogers, L. J., \& Vallortigara, G. (1998). The origins of cerebral asymmetry: A review of evidence of behavioural and brain lateralization in fishes, reptiles and amphibians. Neuroscience and Biobehavioral Reviews, 22, 411-426.

Bódi, N., Kéri, S., Nagy, H., Moustafa, A., Myers, C. E., Daw, N., et al. (2009). Reward-learning and the novelty-seeking personality: A between- and within-subjects study of the effects of dopamine agonists on young Parkinson's patients. Brain, 132, 2385-2395.

Bromberg-Martin, E. S., Matsumoto, M., \& Hikosaka, O. (2010). Dopamine in motivational control: Rewarding, aversive, and alerting. Neuron, 68, 815-834.

Carlson, J. N., \& Stevens, K. D. (2006). Individual differences in ethanol self-administration following withdrawal are associated with asymmetric changes in dopamine and serotonin in the medial prefrontal cortex and amygdala. Alcoholism: Clinical and Experimental Research, 30, 1678-1692.

Carver, C. S., \& White, T. L. (1994). Behavioral inhibition, behavioral activation, and affective responses to impending reward and punishment: The BIS-BAS scales. Journal of Personality and Social Psychology, 67, 310-333.

Cervenka, S., Varrone, A., Fransen, E., Halldin, C., \& Farde, L. (2010). PET studies of D2 receptor binding in striatal and extrastriatal brain regions: Biochemical support in vivo for separate dopaminergic systems in humans. Synapse, 64, 478-485.

Concha, M. L., Biaanco, I. H., \& Wilson, S. W. (2012). Encoding asymmetry within neural circuits. Nature Reviews Neuroscience, 13, 832-843.
Cools, R., Frank, M. J., Gibbs, S. E., Miyakawa, A., Jagust, W., \& D'Esposito, M. (2009). Striatal dopamine predicts outcomespecific reversal learning and its sensitivity to dopaminergic drug administration. Journal of Neuroscience, 29, 1538-1543.

Elliot, A. J. (2008). Approach and avoidance motivation. In A. J. Elliot (Ed.), Handbook of approach and avoidance motivation (pp. 3-14). New York: Psychology Press.

Frank, M. J., \& Fossella, J. A. (2011). Neurogenetics and pharmacology of learning, motivation and cognition. Neuropsychopharmacology, 36, 133-152.

Frank, M. J., Seeberger, L. C., \& O’Reilly, R. C. (2004). By carrot or by stick: Cognitive reinforcement learning in parkinsonism. Science, 306, 1940-1943.

Gray, J. A. (1981). A critique of Eyesenck's theory of personality. In H. J. Eysenck (Ed.), A model for personality (pp. 246-279). Berlin: Springer-Verlag.

Groman, S. M., Lee, B., London, E. D., Mandelkern, M. A., James, A. S., Feiler, K., et al. (2011). Dorsal striatal D2-like receptor availability covaries with sensitivity to positive reinforcement during discrimination learning. Journal of Neuroscience, 31, 7291-7299.

Haber, S. N., Kim, K.-S., Mailly, P., \& Calzavara, R. (2006). Reward-related cortical inputs define a large striatal region in primates that interface with associative cortical connections, providing a substrate for incentive-based learning. Journal of Neuroscience, 26, 8368-8376.

Haber, S. N., \& Knutson, B. (2009). The reward circuit: Linking primate anatomy and human imaging. Neuropsychopharmacol, 35, 4-26.

Hakyemez, H. S., Dagher, A., Smith, S. D., \& Zald, D. H. (2008). Striatal dopamine transmission in healthy humans during a passive monetary reward task. Neuroimage, 39, 2058-2065.

Harmon-Jones, E., Gable, P. A., \& Peterson, C. K. (2010). The role of asymmetric frontal cortical activity in emotionrelated phenomena: A review and update. Biological Psychology, 84, 451-462.

Hietala, J., Syvälahti, E., Vilkman, H., Vuorio, K., Räkköläinen, V., Bergman, J., et al. (1999). Depressive symptoms and presynaptic dopamine function in neuroleptic-naive schizophrenia. Schizophrenia Research, 35, 41-50.

Ichise, M., Toyama, H., Innis, R. B., \& Carson, R. E. (2002). Strategies to improve neuroreceptor parameter estimation by linear regression analysis. Journal of Cerebral Blood Flow $\&$ Metabolism, 22, 1271-1281.

Innis, R. B., Cunningham, V. J., Delforge, J., Fujita, M., Gjedde, A., Gunn, R. N., et al. (2007). Consensus nomenclature for in vivo imaging of reversibly binding radioligands. Journal of Cerebral Blood Flow \& Metabolism, 27, 1533-1539.

Jenkinson, M., \& Smith, S. (2001). A global optimisation method for robust affine registration of brain images. Medical Image Analysis, 5, 143-156.

Jones, A. C., \& Gosling, S. D. (2008). Individual differences in approach and avoidance motivation in animals. In A. J. Elliot (Ed.), Handbook of approach and avoidance motivation (pp. 165-185). New York: Psychology Press.

Laakso, A., Vilkman, H., Alakare, B., Haaoaranta, M., Bergman, J., Solin, O., et al. (2000). Striatal dopamine transporter binding in neuroleptic-nave patients with schizophrenia studies with positron emission tomography. American Journal of Psychiatry, 157, 269-271.

Larisch, R., Meyer, W., Klimke, A., Kehren, F., Vosberg, H., \& Muller-Gartner, H. W. (1998). Left-right asymmetry of striatal dopamine D2 receptors. Nuclear Medicine Communications, 19, 781-787.

Logan, J., Fowler, J. S., Volkow, N. D., Wang, G. J., Ding, Y. S., \& Alexoff, D. L. (1996). Distribution volume ratios without blood sampling from graphical analysis of PET data. Journal of Cerebral Blood Flow \& Metabolism, 16, 834-840. 
Montgomery, A. J., Thielemans, K., Mehta, M. A., Turkheimer, F., Mustafovic, S., \& Grasby, P. M. (2006). Correction of head movement on PET studies: Comparison of methods. Journal of Nuclear Medicine, 47, 1936-1944.

Mukherjee, J., Christian, B. T., Dunigan, K. A., Shi, B., Narayanan, T. K., Satter, M., et al. (2002). Brain imaging of $18 \mathrm{~F}$-fallypride in normal volunteers: Blood analysis, distribution, test-retest studies, and preliminary assessment of sensitivity to aging effects on dopamine D-2/D-3 receptors. Synapse, 46, 170-188.

Mukherjee, J., Yang, Z. Y., Das, M. K., \& Brown, T. (1995). Fluorinated benzamide neuroleptics-III. Development of (S)-N-[(1-allyl-2-pyrrolidinyl)methyl]-5-(3-[18F]fluoropropyl)-2, 3-dimethoxybenzamide as an improved dopamine D-2 receptor tracer. Nuclear Medicine and Biology, 22, 283-296.

Murphy, F. C., Nimmo-Smith, I., \& Lawrence, A. (2003). Functional neuroanatomy of emotions: A meta-analysis. Cognitive, Affective, E Behavioral Neuroscience, 3, 207-233.

Nichols, T. E., \& Holmes, A. P. (2002). Nonparametric permutation tests for functional neuroimaging: A primer with examples. Human Brain Mapping, 15, 1-25.

Porat, O., Hassin-Baer, S., Cohen, O. S., Markus, A., \& Tomer, R. (2013). Asymmetric dopamine loss differentially affects effort to maximize gain or minimize loss. Cortex. dx.doi.org/ 10.1016/j.cortex.2013.10.004.

Ray, N., \& Strafella, A. P. (2010). Dopamine, reward and frontostriatal circuitry in impulse control disorders in Parkinson's disease: Insights from functional imaging. Clinical EEG and Neuroscience, 41, 87-93.

Robbins, T. W., \& Everitt, B. J. (2007). A role for mesencephalic dopamine in activation: Commentary on Berridge (2006). Psychopharmacology, 191, 433-437.

Salamone, J. D., Correa, M., Farrar, A., \& Mingote, S. M. (2007). Effort-related functions of nucleus accumbens dopamine and associated forebrain circuits. Psychopharmacology, 191, 461-482.

Schultz, W. (2007). Behavioral dopamine signals. Trends in Neurosciences, 30, 203-210.

Schutter, D. J., \& Van Honk, D. J. L. D. (2005).

Electrophysiological ratio markers for the balance between reward and punishment. Brain Research. Cognitive Brain Research, 24, 685-690.

Shiner, T., Seymour, B., Wunderlich, K., Hill, C., Bhatia, K. P., Dayan, P., et al. (2012). Dopamine and performance in a reinforcement learning task: Evidence from parkinson's disease. Brain, 135, 1871-1883.

Slagter, H. A., Tomer, R., Christian, B. T., Fox, A. S., Colzato, L. S., King, C. R., et al. (2012). PET evidence for a role for striatal dopamine in the attentional blink: Functional implications. Journal of Cognitive Neuroscience, 24, 1932-1940.

Smittenaar, P., Chase, H. W., Aarts, E., Nusselein, B., Bloem, B. R., \& Cools, R. (2012). Decomposing effects of dopaminergic medication in parkinson's disease on probabilistic action selection-Learning or performance? European Journal of Neuroscience, 35, 1144-1151.

Sutton, S. K., \& Davidson, R. J. (1997). Prefrontal brain asymmetry: A biological substrate of the behavioral approach and inhibition systems. Psychological Science, $8,204-210$.

Thiel, C. M., \& Schwartling, R. K. W. (2001). Dopaminergic lateralization in the forebrain: Relations to behavioral asymmetries and anxiety in male wistar rats. Neuropsychobiology, 43, 192-199.

Toga, A. W., \& Thompson, P. M. (2003). Mapping brain asymmetry. Nature Reviews Neuroscience, 4, 37-48.

Tomer, R., \& Aharon-Peretz, J. (2004). Novelty seeking and harm avoidance in parkinson's disease: Effects of asymmetric dopamine deficiency. Journal of Neurology, Neurosurgery and Psychiatry, 75, 972-975.

Tomer, R., Goldstein, R. Z., Wang, G. J., Wong, C., \& Volkow, N. D. (2008). Incentive motivation is associated with striatal dopamine asymmetry. Biological Psychology, 77, 98-101.

Tomer, R., Slagter, H. A., Christian, B. T., Fox, A. S., King, C. R., Murali, D., et al. (2013). Dopamine asymmetries predict orienting bias in healthy individuals. Cerebral Cortex, 23, 2899-2904.

Trevarthen, C. (1996). Lateral asymmetries in infancy: Implications for the development of the hemispheres. Neuroscience and Biobehavioral Reviews, 20, 571-586.

Van Dyck, C. H., Seibyl, J. P., Malison, R. T., Laruelle, M., Zoghbi, S. S., Baldwin, R. M., et al. (2002). Age related decline in dopamine transporters analysis of striatal subregions, nonlinear effects, and hemispheric asymmetries. American Journal of Geriatric Psychiatry, 10, 36-43.

van Holst, R. J., van den Brink, W., Veltman, D. J., \& Goudiaan, A. E. (2010). Why gamblers fail to win: A review of cognitive and neuroimaging findings in pathological gambling. Neuroscience and Biobehavioral Reviews, 34, 87-107.

Vernaleken, I., Weibrich, C., Siessmeier, T., Buchholz, H. G., Rösch, F., Heinz, A., et al. (2007). Asymmetry in dopamine D $(2 / 3)$ receptors of caudate nucleus is lost with age. Neuroimage, 34, 870-878.

Volkow, N. D., Chang, L., Wang, G.-J., Fowler, J. S., Ding, Y.-S., Sedler, M., et al. (2001). Low level of brain dopamine D2 receptors in methamphetamine abusers: Association with metabolism in the orbitofrontal cortex. American Journal of Psychiatry, 158, 2015-2021.

Wager, T. D., Phan, K. L., Liberzon, I., \& Taylor, S. F. (2003). Valence, gender and lateralization of functional brain anatomy in emotion: A meta-analysis of findings from neuroimaging. Neuroimage, 19, 513-531.

Yacubian, J., \& Buchel, C. (2009). The genetic basis of individual differences in reward processing and the link to addictive behavior and social cognition. Neuroscience, 164, 55-71.

Zald, D. H., Boileau, I., El-Dearedy, W., Gunn, R., McGlone, F., Dichter, G. S., et al. (2004). Dopamine transmission in the human striatum during monetary reward tasks. Journal of Neuroscience, 24, 4105-4112.

Zhang, Y., Brady, M., \& Smith, S. (2001). Segmentation of brain MR images through a hidden Markov random field model and the expectation-maximization algorithm. IEEE Transactions on Medical Imaging, 20, 45-57. 